\title{
The Mass and Current-Meson Coupling Constant of the Exotic X(3872) State from QCD Sum Rules
}

\author{
Hayriye SUNDU *1 \\ ${ }^{1}$ Kocaeli Üniversitesi, Fen ve Edebiyat Fakültesi, Fizik Bölümü, 12345, Kocaeli
}

(Alınış / Received: 05.09.2016, Kabul / Accepted: 11.10.2016, Online Yayınlanma / Published Online: 23.10.2016)

\author{
Keywords \\ Exotic hadron, \\ QCD sum rules, \\ Mass, \\ Current-Meson coupling \\ constant
}

\begin{abstract}
The mass and current-meson coupling constant of the exotic $X(3872)$ state are computed within the two-point sum rule method using the diquark-antidiquark and molecule interpolating currents. In calculations the quark, gluon and mixed vacuum condensates up to ten dimensions are taken into account. Within the diquark-antidiquark picture for the mass of the $X(3872)$ state we obtain $m_{X}=3873 \mathrm{MeV}$, which is very close to the experimental result of the Belle Collaboration. The molecule model leads for $m_{X}$ to the prediction that slightly exceeds the experimental data. We compare our predictions for the mass and coupling constant with available experimental data and other theoretical results.
\end{abstract}

\section{KRD Toplam Kurallarında Ekzotik X(3872) Durumunun Kütle ve Akım-Mezon Kuplaj Sabiti}

Anahtar Kelimeler

Ekzotik hadron,

KRD toplam kuralları,

Kütle,

Akım-Mezon kuplaj sabiti

\begin{abstract}
Özet: Ekzotik $X(3872)$ durumunun kütle ve akım-mezon kuplaj sabiti, dikuarkantidikuark ve molekül arakesit akımları kullanılarak iki-nokta toplam kuralları metodu ile hesaplanmıştır. Hesaplamalarda, on boyuta kadar kuark, gluon ve birleşik vakum kondensatları göz önüne alınmıştır. Dikuark-antidikuark akım çerçevesinde $X(3872)$ durumunun kütlesi için, Belle İşbirliğinin deneysel sonucu ile oldukça yakın olan $m_{X}=$ $3873 \mathrm{MeV}$ değeri elde edilmiştir. Molekül model, $m_{X}$ öngörüsü için deneysel sonucu kısmen aşı̆̆ı̆ı ileri sürmektedir. Kütle ve kuplaj sabiti için elde edilen sonuçlar deney dataları ve diğer teorik sonuçlar ile karşılaştırılmıştır.
\end{abstract}

\section{Introduction}

During last decade due to an experimental information of the Belle, BaBar, BESIII, LHCb, CDF and some other collaborations, investigation of exotic states, i.e. states that cannot be included into the quark-antiquark and three-quark schemes of the traditional hadron spectroscopy, became one of the interesting and growing fields in the hadron physics.

The productive period of intensive experimental and theoretical studies of exotic particles started from the discovery of the charmonium-like resonance $X(3872)$ Belle Collaboration [1]. confirmed later in some other experiments [2-4]. The exotic states were produced and observed in $B$ meson decays, in the $e^{+} e^{-}$and $\bar{p} p$ annihilations, in the double charmonium production processes, in the two-photon fusion and $p p$ collisions. Experimental investigations encompass measurements of the masses and decay widths of these states, exploration of their spins, parities and C-parities.

Recently, the D0 Collaboration reported on the observation of the resonance $X(5568)$ in [5]. The new particle
$X(5568)$ contains four quarks of the different flavors $b, s, u, d$ and belongs to the class of the exotic states. The $X(5568)$ is supposedly the first exotic particle composed of four-quarks of the different flavors, which makes it a very interesting object for both the experimental and theoretical studies. The D0 Collaboration measured its mass, width of the dominant decay channel $X(5568) \rightarrow B_{S} \pi$, and studied its quantum numbers considering $J^{P C}=0^{++}$ as preferable ones. The observed and studied exotic states are collected into the $X Y Z$ family of new particles (see, the reviews [6-13] and references therein).

These discoveries necessitated generating of new theoretical approaches to interpret underlying quark-gluon structure of the exotic states, and inventing methods for calculation of their properties. Thus, efforts were done to consider charmonium-like resonances as excitations of the ordinary $c \bar{c}$ charmonium. It should be noted that some of them really allow such interpretation. But the main part of the collected experimental data cannot be included into this frame, and therefore for understanding of the phenomenology of $X Y Z$ states various quark-gluon models were suggested. 
One of the mostly employed models is the four-quark or tetraquark picture of the exotic states. These quarks may cluster into colored diquarks and antidiquarks, which are organized in such a way that to reproduce quantum numbers of the corresponding exotic states, and is known as the diquark- antidiquark model [14]. In the meson-molecule model the exotic particle may appear as a bound state of two color-singlet mesons. These two models were widely used to explore features of the exotic states. Thus, in theoretical studies aimed to explore the exotic $X(5568)$ state and calculate its parameters both of these models were employed [15-17]. Investigation of the new family of the four-quark states was extended in [18] by considering a charmed partner of $X(5568)$, i.e. the state $X_{c}$ containing $c, s, u, d$ quarks and possessing the same quantum numbers as the $X(5568)$ state. The mass and coupling constant of $X_{c}$ were computed within the diquark-antidiquark model by applying two different interpolating currents.

As it has just been emphasized, $X(3872$ ) (in what follows denoted also as the $X$ ) resonance is the first discovered and, therefore studied exotic state of the $X Y Z$ family. It was detected in $B$ meson decay $B^{+} \rightarrow X(3872) K^{+} \rightarrow J / \psi \pi^{+} \pi^{-} K^{+}$by Belle Collaboration [1]. Later the $X(3872)$ was observed also through direct production mode in $\bar{p} p$ annihilation [2, 3], as well as in the $e^{+} e^{-}$annihilation and $p p$ collisions [13]. The assigned quantum numbers to $X$ state are $1^{++}$, its average mass extracted from different experiments is $m_{X}=(3871.69 \pm 0.17) \mathrm{MeV}$, and the decay width was estimated as $\Gamma<1.2 \mathrm{MeV}[10,19,20]$. The collected data allowed one to make some suggestions on the possible quark-antiquark structure of the $X$ state. In fact, its mass is close to the $D \overline{D^{*}}$ threshold, therefore $X$ can be considered as a state built of these mesons. Another possibility is accepting $X$ as an admixture of the molecule and charmonium components.

In the present work we compute the mass and coupling constant of the $X$ state within the QCD two-point sum rule method. Similar calculations of the $X$ state parameters were performed in Refs. [21-24], as well. In [21] the authors considered this particle as a diquark-antidiquark state within the tetraquark model of the exotic mesons. Calculations were performed by taking into account part of the quark, gluon and mixed condensates up to dimension eight. In [22] the authors considered this particle as a mixed molecule-charmonium state, and again calculated its mass with the same accuracy. In other words, in both cases they ignored the contribution coming from the condensates with dimension nine and dimension ten. The width of the radiative decay of the meson $X$ was calculated assumed to be a mixture between charmonium and exotic molecule states in [23]. In [24] the $X$ was modeled as only axial-vector diquark-antidiquark state. In this work we present two different calculations for the mass and coupling constant of the exotic $X$ state by applying two different models that assume this state as a diquarkantidiquark and molecule states. We carried out the com- putation of parameters of the $X$ state by including vacuum condensates up to dimension ten, which improves the sum rule increasing its stability.

\section{Material and Method}

To calculate the mass and coupling constant of the $X$ state in the framework of QCD sum rules, we start from the two-point correlation function

$$
\Pi_{\mu v}(q)=i \int d^{4} x e^{i q \cdot x}\left\langle 0\left|\mathscr{T}\left\{J_{\mu}(x) J_{v}^{\dagger}(0)\right\}\right| 0\right\rangle,
$$

where $J_{\mu}(x)$ is the interpolating current with required quantum numbers. We consider $X(3872)$ state as a particle with the quantum numbers $J^{P C}=1^{++}$. Then in the diquark model the current $J_{\mu}^{(\mathrm{Di})}(x)$ is defined by the following expression [21]

$$
\begin{aligned}
& J_{\mu}^{\mathrm{Di}}(x)=\frac{i \varepsilon \tilde{\varepsilon}}{\sqrt{2}}\left\{\left[q_{a}^{T}(x) C \gamma_{5} c_{b}(x)\right]\left[\bar{q}_{d}(x) \gamma_{\mu} C \bar{c}_{e}^{T}(x)\right]\right. \\
& \left.+\left[q_{a}^{T}(x) C \gamma_{\mu} c_{b}(x)\right]\left[\bar{q}_{d}(x) \gamma_{5} C \bar{c}_{e}^{T}(x)\right]\right\}
\end{aligned}
$$

where $q$ is one of the light $u$ or $d$ quarks. Here we have introduced the short-hand notations $\varepsilon=\varepsilon_{a b c}$ and $\tilde{\varepsilon}=\varepsilon_{d e c}$. In Eq. (2) $a, b, c, d, e$ are color indexes and $C$ is the charge conjugation matrix. One can also construct the interpolating current with required quantum numbers by considering $X$ as a molecular $D \bar{D}^{*}$ state [22]

$$
\begin{aligned}
J_{\mu}^{\mathrm{Mol}}(x)= & \frac{1}{\sqrt{2}}\left\{\left[\bar{q}_{a}(x) \gamma_{5} c_{a}(x)\right]\left[\bar{c}_{b}(x) \gamma_{\mu} c_{b}(x)\right]\right. \\
& \left.-\left[\bar{q}_{a}(x) \gamma_{\mu} c_{a}(x)\right]\left[\bar{c}_{b}(x) \gamma_{5} c_{b}(x)\right]\right\} .
\end{aligned}
$$

In order to derive QCD sum rule expression we first calculate the correlation function in terms of the physical degrees of freedom. Performing integral over $x$ in Eq. (1), we get

$$
\Pi_{\mu \nu}^{\text {Phys }}(q)=\frac{\left\langle 0\left|J_{\mu}\right| X(q)\right\rangle\left\langle X(q)\left|J_{v}^{\dagger}\right| 0\right\rangle}{m_{X}^{2}-q^{2}}+\ldots
$$

where $m_{X}$ is the mass of $X$ state, and dots stand for contributions of the higher resonances and continuum states. We define the decay constant $f_{X}$ through the matrix element

$$
\left\langle 0\left|J_{\mu}\right| X(q)\right\rangle=f_{X} m_{X} \varepsilon_{\mu},
$$

with $\varepsilon_{\mu}$ being the polarization vector of $X$ state. Then in terms of $m_{X}$ and $f_{X}$, the correlation function can be written in the form

$$
\Pi_{\mu v}^{\text {Phys }}(q)=\frac{m_{X}^{2} f_{X}^{2}}{m_{X}^{2}-q^{2}}\left(-g_{\mu v}+\frac{q_{\mu} q_{v}}{m_{X}^{2}}\right)+\ldots
$$

The Eq. (5) is obtained by inserting into $\Pi_{\mu v}^{\text {Phys }}(q)$ intermediate states for $X$ and projecting out the $1^{++}$contribution by means of the relevant Lorentz structure. Here we use also the single pole assumption, which in the case of a multiquark state should be additionally justified. The reason is that for such systems in the physical side of the sum rules one has to take into account also two-hadron reducible contribution [25]. But in our case, as it 
was demonstrated in Ref. [22], due to smallness of the coupling constant $\lambda_{D D^{*}}$ this contribution can be neglected.

The Borel transformation applied to Eq. (5) yields

$$
\mathscr{B}_{q^{2}} \Pi_{\mu \nu}^{\mathrm{Phys}}(q)=m_{X}^{2} f_{X}^{2} e^{-m_{X}^{2} / M^{2}}\left(-g_{\mu v}+\frac{q_{\mu} q_{v}}{m_{X}^{2}}\right)+\ldots
$$

It should be noted that the general expressions given by Eqs. (5) and (6) are valid for both the diquark-antidiquark and molecular currents.

The same function in QCD side, $\Pi_{\mu v}^{\mathrm{QCD}}(q)$, has to be determined employing of the quark-gluon degrees of freedom. To this end, we contract the heavy and light quark fields and find for the correlation function $\Pi_{\mu \nu}^{\mathrm{QCD}(\mathrm{Di})}(q)$ in the diquark picture the following expression:

$$
\begin{aligned}
& \Pi_{\mu v}^{\mathrm{QCD}(\mathrm{Di})}(q)=-\frac{i}{2} \int d^{4} x e^{i q x} \varepsilon \tilde{\varepsilon} \varepsilon^{\prime^{\prime}} \tilde{\varepsilon}^{\prime}\left\{\operatorname { T r } \left[\gamma_{5} \widetilde{S}_{q}^{a a^{\prime}}(x)\right.\right. \\
& \left.\times \gamma_{5} S_{c}^{b b^{\prime}}(x)\right] \operatorname{Tr}\left[\gamma_{\mu} \widetilde{S}_{c}^{e^{\prime} e}(-x) \gamma_{v} S_{q}^{d^{\prime} d}(-x)\right] \\
& +\operatorname{Tr}\left[\gamma_{\mu} \widetilde{S}_{c}^{e^{\prime} e}(-x) \gamma_{5} S_{q}^{d^{\prime} d}(-x)\right] \operatorname{Tr}\left[\gamma_{v} \widetilde{S}_{q}^{a a^{\prime}}(x)\right. \\
& \left.\times \gamma_{5} S_{c}^{b b^{\prime}}(x)\right]+\operatorname{Tr}\left[\gamma_{5} \widetilde{S}_{q}^{a^{\prime} a}(x) \gamma_{\mu} S_{c}^{b^{\prime} b}(x)\right] \\
& \times \operatorname{Tr}\left[\gamma_{5} \widetilde{S}_{c}^{e^{\prime} e}(-x) \gamma_{v} S_{q}^{d^{\prime} d}(-x)\right]+\operatorname{Tr}\left[\gamma_{v} \widetilde{S}_{q}^{a a^{\prime}}(x)\right. \\
& \left.\left.\times \gamma_{\mu} S_{c}^{b b^{\prime}}(x)\right] \operatorname{Tr}\left[\gamma_{5} \widetilde{S}_{c}^{e^{\prime} e}(-x) \gamma_{5} S_{q}^{d^{\prime} d}(-x)\right]\right\}
\end{aligned}
$$

The correlation function $\Pi_{\mu v}^{\mathrm{QCD}(M o l)}(q)$ in the molecular model reads

$$
\begin{aligned}
& \Pi_{\mu v}^{\mathrm{QCD}(\mathrm{Mol})}(q)=-\frac{i}{2} \int d^{4} x e^{i q x}\left\{\operatorname { T r } \left[\gamma_{5} S_{c}^{a a^{\prime}}(x)\right.\right. \\
& \left.\times \gamma_{5} S_{q}^{a^{\prime} a}(-x)\right] \operatorname{Tr}\left[\gamma_{\mu} S_{q}^{b b^{\prime}}(x) \gamma_{v} S_{c}^{b^{\prime} b}(-x)\right] \\
& -\operatorname{Tr}\left[\gamma_{5} S_{c}^{a a^{\prime}}(x) \gamma_{v} S_{q}^{a^{\prime} a}(-x)\right] \operatorname{Tr}\left[\gamma_{\mu} S_{q}^{b b^{\prime}}(x)\right. \\
& \left.\times \gamma_{5} S_{c}^{b^{\prime} b}(-x)\right]-\operatorname{Tr}\left[\gamma_{\mu} S_{c}^{a a^{\prime}}(x) \gamma_{5} S_{q}^{a^{\prime} a}(-x)\right] \\
& \times \operatorname{Tr}\left[\gamma_{5} S_{q}^{b b^{\prime}}(x) \gamma_{v} S_{c}^{b^{\prime} b}(-x)\right]+\operatorname{Tr}\left[\gamma_{\mu} S_{c}^{a a^{\prime}}(x)\right. \\
& \left.\left.\times \gamma_{v} S_{q}^{a^{\prime} a}(-x)\right] \operatorname{Tr}\left[\gamma_{5} S_{q}^{b b^{\prime}}(x) \gamma_{5} S_{c}^{b^{\prime} b}(-x)\right]\right\}
\end{aligned}
$$

In Eq. (7) we use the notation

$$
\widetilde{S}_{c(q)}^{i j}(x)=C S_{c(q)}^{i j T}(x) C,
$$

with $S_{q}^{i j}(x)$ and $S_{c}^{i j}(x)$ being the light and heavy quark propagators, respectively. Neglecting terms $\sim m_{q}$, we choose the light quark propagator $S_{q}^{i j}(x)$ in the $x$-space in the form

$$
\begin{aligned}
& S_{q}^{i j}(x)=i \delta_{i j} \frac{\not x}{2 \pi^{2} x^{4}}-\delta_{i j} \frac{\langle\bar{q} q\rangle}{12}-\delta_{i j} \frac{x^{2}}{192}\langle\bar{q} g \sigma G q\rangle \\
& -i \frac{g G_{i j}^{\alpha \beta}}{32 \pi^{2} x^{2}}\left[/ x \sigma_{\alpha \beta}+\sigma_{\alpha \beta} / x\right]-i \delta_{i j} \frac{x^{2} / x g^{2}\langle\bar{q} q\rangle^{2}}{7776} \\
& -\delta_{i j} \frac{x^{4}\langle\bar{q} q\rangle\left\langle g^{2} G G\right\rangle}{27648}+\ldots
\end{aligned}
$$

For the heavy quark propagator $S_{c}^{i j}(x)$ we employ the expression [26]

$$
\begin{gathered}
S_{c}^{i j}(x)=i \int \frac{d^{4} k}{(2 \pi)^{4}} e^{-i k x}\left[\frac{\delta_{i j}\left(\not k+m_{c}\right)}{k^{2}-m_{c}^{2}}\right. \\
-\frac{g G_{i j}^{\alpha \beta}}{4} \frac{\sigma_{\alpha \beta}\left(\not k+m_{c}\right)+\left(\not k+m_{c}\right) \sigma_{\alpha \beta}}{\left(k^{2}-m_{c}^{2}\right)^{2}} \\
\left.+\frac{g^{2}}{12} G_{\alpha \beta}^{a} G^{a \alpha \beta} \delta_{i j} m_{c} \frac{k^{2}+m_{c} \not k}{\left(k^{2}-m_{c}^{2}\right)^{4}}+\ldots\right] .
\end{gathered}
$$

In the above the short-hand notation

$$
G_{i j}^{\alpha \beta} \equiv G_{a}^{\alpha \beta} t_{i j}^{a}, a=1,2 \ldots 8,
$$

is used, where $i, j$ are color indexes, and $t^{a}=\lambda^{a} / 2$, where $\lambda^{a}$ being the standard Gell-Mann matrices. The first term in Eq. (10) is the free massive quark propagator, next ones are nonperturbative gluon corrections. In the nonperturbative terms the gluon field strength tensor $G_{\alpha \beta}^{a} \equiv G_{\alpha \beta}^{a}(0)$ is fixed at $x=0$.

The correlation function $\Pi_{\mu \nu}^{\mathrm{QCD}}(q)$ can be decomposed over the Lorentz structures

$\Pi_{\mu \nu}^{\mathrm{QCD}}(q)=\Pi_{0}^{\mathrm{QCD}}\left(q^{2}\right) \frac{q_{\mu} q_{v}}{q^{2}}+\Pi_{1}^{\mathrm{QCD}}\left(q^{2}\right)\left(-g_{\mu \nu}+\frac{q_{\mu} q_{v}}{q^{2}}\right)$,

where the first term is the contribution of spin 0 mesons, whereas the second function $\Pi_{1}^{\mathrm{QCD}}\left(q^{2}\right)$ corresponds to spin 1 mesons. The QCD sum rule expressions for the mass and coupling constant are derived after fixing the same structures in both $\Pi_{\mu \nu}^{\mathrm{Phys}}(q)$ and $\Pi_{\mu \nu}^{\mathrm{QCD}}(q)$. To exclude effects of spin 0 particles, we work with the term proportional to $g_{\mu \nu}$. Denoting $\widetilde{\Pi}^{\mathrm{QCD}}\left(q^{2}\right)=-\Pi_{1}^{\mathrm{QCD}}\left(q^{2}\right)$, we can write it as the dispersion integral,

$$
\widetilde{\Pi}^{\mathrm{QCD}}\left(q^{2}\right)=\int_{4 m_{c}^{2}}^{\infty} \frac{\rho^{\mathrm{QCD}}(s)}{s-q^{2}}+\ldots
$$

where $\rho^{\mathrm{QCD}}(s)$ is the corresponding spectral density.

The main question of this section is calculation of $\rho_{\mathrm{Di}}^{\mathrm{QCD}}(s)$ and $\rho_{\mathrm{Mol}}^{\mathrm{QCD}}(s)$. In the present work we include into our sum rules the quark, gluon and mixed condensates up to ten dimensions. For computation of the components of the spectral densities we use the technical methods outlined in Ref. [27]. Nevertheless, for completeness of the presentation we provide essential stages and relevant formulas for their realization. We first apply the transformation

$$
\begin{aligned}
& \frac{1}{\left(x^{2}\right)^{n}}=\int \frac{d^{D} t}{(2 \pi)^{D}} e^{-i t \cdot x} i(-1)^{n+1} 2^{D-2 n} \pi^{D / 2} \\
& \times \frac{\Gamma(D / 2-n)}{\Gamma(n)}\left(-\frac{1}{t^{2}}\right)^{D / 2-n},
\end{aligned}
$$

and replace, where necessary $x_{\mu}$ by $-i \partial / \partial q_{\mu}$, and calculate the $x$ integral. As a result, we obtain the delta function with a combination of the momenta in its argument. This Dirac delta is used later to remove one of the momentum integrals. The remaining integrations over $t$ and $k$ require invoking the Feynman parametrization and performing rearrangements of denominators in obtained expressions. After that we carry out integration over $t$ and 
perform the last integral over $k$ by means of the formulas

$$
\int d^{4} k \frac{1}{\left(k^{2}+L\right)^{\alpha}}=\frac{i \pi^{2}(-1)^{\alpha} \Gamma(\alpha-2)}{\Gamma(\alpha)[-L]^{\alpha-2}}, .
$$

The imaginary part of the correlation function can now be extracted by applying in the $D \rightarrow 4$ limit the replacement

$$
\Gamma\left(\frac{D}{2}-n\right)\left(-\frac{1}{L}\right)^{\frac{D}{2}-n} \rightarrow \frac{(-1)^{n-1}}{(n-2) !}(-L)^{n-2} \ln (-L)
$$

or the well known formula

$$
\frac{1}{s-A}=\mathrm{P} . \mathrm{V} \cdot \frac{1}{s-A}-i \pi \delta(s-A) .
$$

Results of our calculations performed in accordance with this recipe are collected in Appendix A.

Applying the Borel transformation on the variable $q^{2}$ to the invariant amplitude $\widetilde{\Pi}^{\mathrm{QCD}}\left(q^{2}\right)$, equating the obtained expression with the relevant part of $\mathscr{B}_{q^{2}} \Pi_{\mu \nu}^{\text {Phys }}(q)$, and subtracting the continuum contribution, we finally obtain the required sum rule. Thus, the mass of the $X$ state can be evaluated from the sum rule

$$
m_{X}^{2}=\frac{\int_{4 m_{c}^{2}}^{s_{0}} d s s \rho^{\mathrm{QCD}}(s) e^{-s / M^{2}}}{\int_{4 m_{c}^{2}}^{s_{0}} d s \rho(s) e^{-s / M^{2}}}
$$

whereas to extract the numerical value of the decay constant $f_{X}$ we employ the formula

$$
f_{X}^{2} e^{-m_{X}^{2} / M^{2}}=\frac{1}{m_{X}^{2}} \int_{4 m_{c}^{2}}^{s_{0}} d s \rho^{\mathrm{QCD}}(s) e^{-s / M^{2}} .
$$

The last two equations are the sum rules required to evaluate the $X$ state's mass and decay constant, respectively.

\section{Numeric Results}

The QCD sum rules for the mass and coupling constant of the $X$ state contain as parameters various quark, gluon and mixed vacuum condensates. Their values are collected in Table 1

Table 1. Values of the vacuum condensates used in numerical calculations.

\begin{tabular}{|c|c|c|}
\hline Parameters & Value & Unit \\
\hline$\langle\bar{q} q\rangle$ & $(-0.24 \pm 0.01)^{3}$ & {$\left[\mathrm{GeV}^{3}\right]$} \\
\hline$\left\langle\frac{\alpha_{s} G^{2}}{\pi}\right\rangle$ & $(0.012 \pm 0.004)$ & {$\left[\mathrm{GeV}^{4}\right]$} \\
\hline$m_{0}^{2}$ & $(0.8 \pm 0.1)$ & {$\left[\mathrm{GeV}^{3}\right]$} \\
\hline$\alpha_{s}$ & $0.1185(6)$ & - \\
\hline
\end{tabular}

The QCD sum rule' expressions depend also on the continuum threshold and Borel parameters, i.e. on $s_{0}$ and $M^{2}$, respectively. We have to determine some regions where physical quantities in question are almost independent on $s_{0}$ and $M^{2}$, or demonstrate unessential dependence on them. In order to fix the working window for the Borel parameter, we require the convergence of the OPE, as well as suppression of the contributions arising from the higher resonances and continuum, in other words exceeding of the pole contribution over the ones coming from the higher dimensional condensates. As a result, for the mass and coupling constant calculations we find the range of $M^{2}$

$$
2.5 \mathrm{GeV}^{2} \leq M^{2} \leq 5.0 \mathrm{GeV}^{2},
$$

as a reliable for our purposes. It is worth noting that in this interval the dependence of the mass and decay constant on mass $M^{2}$ is stable, and we may expect that the sum rules give the correct results.

It is known that, the continuum threshold $s_{0}$ depends on the mass of the first excited state with the same quantum numbers and structure as the particle in question. In the case of the exotic particles, it is difficult to fix unambiguously this exited state, therefore we have to follow usual prescriptions accepted in the sum rules calculations and fix $s_{0}$ as $s_{0}=\left(m_{X}+[0.3 \div 0.5]\right)^{2}$, i.e.

$$
17.41 \mathrm{GeV}^{2} \leq s_{0} \leq 19.11 \mathrm{GeV}^{2}
$$

The QCD sum rules suffer from uncertainties inherent to the method itself. The main sources of the theoretical errors are the Borel and continuum threshold parameters. By varying the parameters $M^{2}$ and $s_{0}$ within the allowed ranges, as well as taking into account ambiguities arising from other input parameters and adding relevant uncertainties in the quadrature, we estimate total errors of the computed quantities. The results for the mass $m_{X}$ and coupling constant $f_{X}$ obtained using the diquark-antidiquark model are drawn in Figure 1 and Figure 2, whereas in Figure 3 and Figure 4 we show the same quantities in the case of the molecule model. Our results for the mass and coupling constant of the exotic $X(3872)$ state are collected in Table 2.

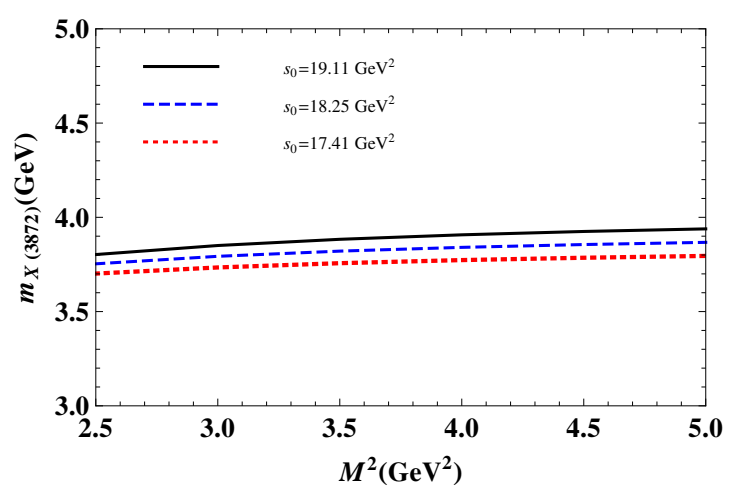

Figure 1. The diquark-antidiquark model: the mass $m_{X}$ as a function of the Borel parameter $M^{2}$ for different values of $s_{0}$.

As is seen, the prediction for the mass $m_{X}$ obtained using the molecule model is larger than the same quantity extracted within the diquark-antidiquark picture. At the same time, we observe the opposite situation with the coupling constant $f_{X}$. Nevertheless, both of them, within the errors, agree with each other. The obtained the predictions for the mass $m_{X}$ are compatible with the experimental result

$$
m_{X}=(3871.69 \pm 0.17) \mathrm{MeV} .
$$

The spectroscopic parameters of the $X$ state were calculated in Refs. [21-24], as well. Thus, in the work 
Table 2. The sum rule results for the $m_{X}$ and $f_{X}$.

\begin{tabular}{|c|c|c|c|}
\hline & $J_{\mu}^{D i}$ & $J_{\mu}^{M o l}$ & Unit \\
\hline$m_{X}$ & $3873 \pm 127$ & $3908 \pm 139$ & {$[\mathrm{MeV}]$} \\
\hline$f_{X}$ & $(0.56 \pm 0.19) \times 10^{-2}$ & $(0.57 \pm 0.21) \times 10^{-2}$ & {$\left[\mathrm{GeV}^{4}\right]$} \\
\hline
\end{tabular}

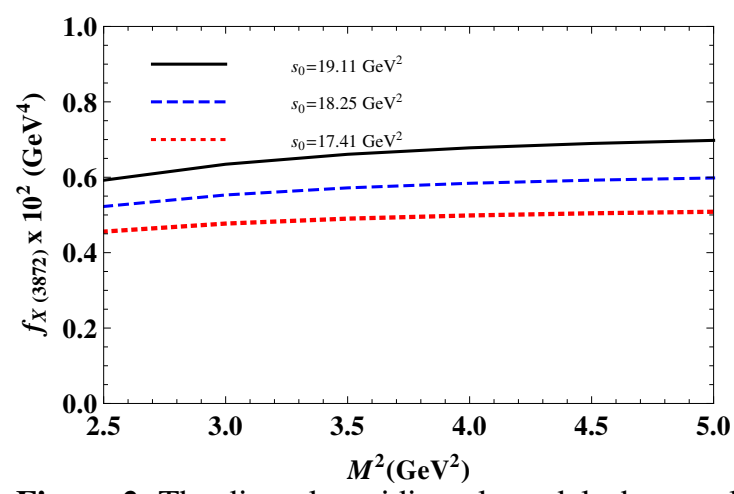

Figure 2. The diquark-antidiquark model: the coupling constant $f_{X}$ vs Borel parameter $M^{2}$.

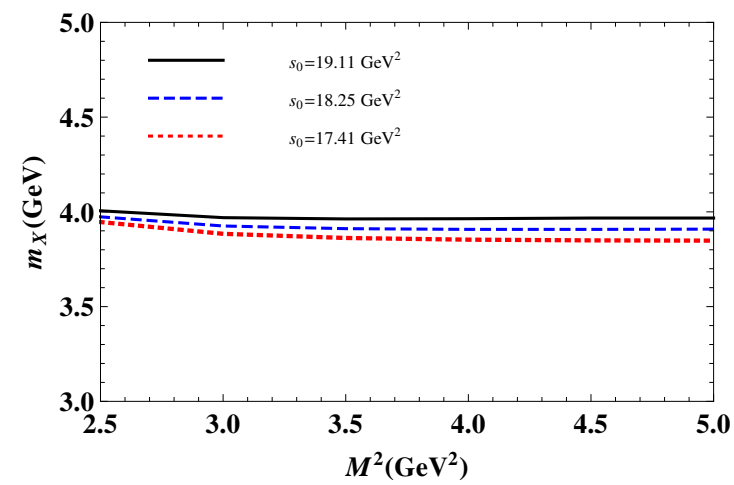

Figure 3. The molecule model: the mass $m_{X}$ as a function of the Borel parameter $M^{2}$ for different values of $s_{0}$.

Ref. [21] the $X(3872)$ particle was considered as the diquark-antidiquark exotic state with quantum numbers $J^{P C}=1^{++}$. The mass of the particle was evaluated using the two-point QCD sum rule method, and part of vacuum condensates up to dimension eight were taken into account. The result obtained for the mass $m_{X}$ of the $X$ state is:

$$
m_{X}=(3925 \pm 127) \mathrm{MeV} .
$$

The similar computations were performed in Ref. [22], where the state was analyzed as an admixture of the molecule and charmonium components. The authors carried out computations again by taking into account condensates up to dimension eight and found

$$
m_{X}=(3770 \pm 180) \mathrm{MeV} .
$$

In Ref. [24] the $X$ was modeled as axial-vector diquarkantidiquark state, where for its mass the following result was obtained:

$$
m_{X}=(3870 \pm 90) \mathrm{MeV}
$$

As is seen, our result for the mass of the $X$ state extracted from QCD two-point sum rules are in agreement with the

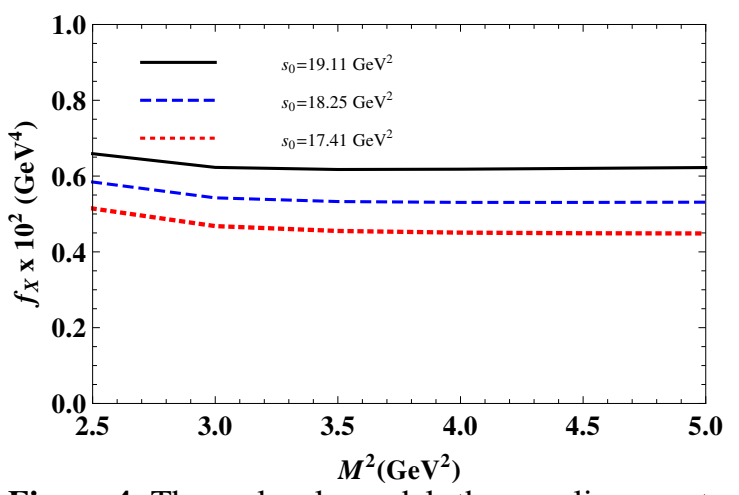

Figure 4. The molecule model: the coupling constant $f_{X}$ vs Borel parameter $M^{2}$.

available theoretical predictions, if take into account the errors, which are unavoidable part of such kind of calculations.

\section{Discussion and Conclusion}

In this work we have calculated the mass and coupling constant of the exotic $X(3872)$ by modelling it as the diquark-antidiquark and molecule states. In computations we have employed QCD two-point sum rule method and take into account vacuum condensates up to dimension ten. We have found that for $m_{X}$ the diquark-antidiquark interpolating current leads to lower value than the molecule model, whereas for the coupling constant $f_{X}$ we have observed the opposite situation. Our results agree, within the errors, with the experimental data. They are also in accord with available theoretical predictions. It is clear also, that computation only of the mass and coupling constant does not allow us to make a final conclusion on the internal structure of the $X(3872)$ state. This aim may be achieved by including into analysis the vacuum condensates of more than ten dimensions, as well as by exploring various decay channels of the $X(3872)$ state, which are more sensitive to its internal organization.

\section{Acknowledgment}

The author is grateful to S. Agaev and K. Azizi for helpful discussions.

\section{References}

[1] Choi, S.-K., et al. (Belle Collaboration) 2003. Observation of a Narrow Charmoniumlike State in Exclusive $B^{ \pm} \rightarrow K^{ \pm} \pi^{+} \pi^{-} J / \psi$ Decays. Physical Review Letters, 91(2003), 262001.

[2] Abazov, V. M., et al. (D0 Collaboration) 2004. Observation and Properties of the $X(3872)$ Decaying to $J / \psi \pi^{+} \pi^{-}$in $p \bar{p}$ Collisions at $\sqrt{s}=1.96 \mathrm{TeV}$. Physical Review Letters, 93(2004), 162002. 
[3] Acosta, D., et al. (CDF II Collaboration) 2004. Observation of the Narrow State $X(3872) \rightarrow J / \psi \pi^{+} \pi^{-}$ in $p \bar{p}$ Collisions at $\sqrt{s}=1.96 \mathrm{TeV}$. Physical Review Letters, 93(2004), 072001.

[4] Aubert, B., et al. (BaBar Collaboration) 2005. Study of the $B^{-} \rightarrow J / \psi K^{-} \pi^{+} \pi^{-}$decay and measurement of the $B^{-} \rightarrow X(3872) K^{-}$branching fraction. Physical Review D, 71(2005), 071103.

[5] Abazov, V. M., et al. (D0 Collaboration) 2016. Evidence for a $B_{s}^{0} \pi^{ \pm}$State. Physical Review Letters, 117(2016), 022003.

[6] Swanson, E. S. 2006. The New Heavy Mesons: A status Report. Physics Reports, 429(2006), 243-305.

[7] Klempt, E., Zaitsev, A. 2007. Glueballs, hybrids, multiquarks: Experimental facts versus QCD inspires concept. Physics Reports, 454(2007), 1-202.

[8] Godfrey, S., Olsen, S. L. 2008. The Exotic $X Y Z$ Charmonium-Like Mesons. Annual Review of $\mathrm{Nu}-$ clear and Particle Science, 58(2008), 51-73.

[9] Voloshin, M. B. 2008. Charmonium. Progress in Particle and Nuclear Physics, 61(2008), 455-511.

[10] Nielsen, M., Navarra F. S., Lee S. H. 2010. New Charmonium States in QCD Sum Rules: a Concise Review. Physics Reports, 497(2010), 41-83.

[11] Faccini, R., Pilloni, A., Polosa, A. D. 2012. Exotic Heavy Quarkonium Spectroscopy: A mini-review. Modern Physics Letters A, 27(2012), 1230025.

[12] Esposito, A. Guerrieri, A. L., Piccinini, F., Pilloni, A., Polosa, A. D. 2014. Four-quark hadrons: An updated review. International Journal of Modern Physics A, 30(2014), 1530002.

[13] Chen, H.-X., Chen, W., Liu, X., Zhu, S.-L. 2016. The hidden-charm pentaquark and teraquark states. Physics Reports, 639(2016), 1-121.

[14] Maiani, L., Piccinini, F., Polosa, A. D., Riquer, V. 2005. Diquark-antiquark states with hidden or open charm and the nature of $X(3872)$. Physical Review D, 71(2005), 014028.

[15] Agaev, S. S., Azizi, K., Sundu, H. 2016. Mass and decay constant of the newly observed exotic $X(5568)$ state .Physical Review D, 93(2016), 074024.

[16] Agaev, S. S., Azizi, K., Sundu, H. 2016. Width of the exotic $X_{b}(5568)$ state through its strong decay to $B_{s}^{0} \pi^{+}$. Physical Review D, 93(2016), 114007.

[17] Agaev, S. S., Azizi, K., Sundu, H. 2016. Exploring $X(5568)$ as a meson molecule. The European Physical Journal Plus , 131(2016), 351.

[18] Agaev, S. S., Azizi, K., Sundu, H. 2016. Charmed partner of the exotic $X(5568)$ stateand its properties. Physical Review D, 93(2016), 094006.

[19] Olive, K. A., et al. [Particle Data Group Collaboration] 2014. Review of Particle Physics. Chinese Physics C, 38(2014), 090001.
[20] Choi, S.-K., et al. 2011. Bounds on the width, mass difference and other properties of $X(3872) \rightarrow$ $\pi^{+} \pi^{-} J / \psi$ decays. Physical Review D, 84(2011), 052004 .

[21] Matheus, R. D., Narison, S., Nielsen M., Richard, J. M. 2007. Can the $X(3872)$ be a $1^{++}$four-quark state? Physical Review D, 75(2007), 014005.

[22] Matheus, R. D., Navarra, F. S., Nielsen, M., Zanetti, C. M. 2009. QCD sum rules for the $X(3872)$ as a mixed molecule-charmonium state. Physical Review D, 80(2009), 056002.

[23] Nielsen, M., Zanetti, C. M. 2010. Radiative decay of the $X(3872)$ as a mixed molecule-charmonium state in QCD sum rules. Physical Review D, 82(2010), 116002.

[24] Wang, Z. G., Huang, T. 2014. Analysis of the $X(3872), Z_{c}(3900)$, and $Z_{c}(3885)$ as axial-vector tetraquarks states with QCD sum rules. Physical Review D, 89(2014), 054019.

[25] Kondo, Y., Morimatsu, O., Nishikawa, T. 2005. Teo-hadron-irreducible QCD sum rule for pentaquark baryon. Physics Letters B, 611(2005), 93-101.

[26] Reinders, L. J., Rubinstein, H., Yazaki, S. 1985. Hadron properties from QCD sum rules. Physics Reports, 127(1985), 1-97.

[27] Agaev, S. S., Azizi, K., Sundu, H. 2016. Strong $Z_{c}^{+}(3900) \rightarrow J / \psi \pi^{+} ; \eta_{c} \rho^{+}$decays in QCD. Physical Review D, 93(2016), 074002.

\section{Appendix}

In this appendix we have collected the results of our calculations of the spectral density

$$
\rho^{Q C D}(s)=\rho^{\text {pert }}(s)+\sum_{k=3}^{k=10} \rho_{k}(s),
$$

necessary for evaluation of the mass and coupling constant of the $X(3872)$ state from the QCD sum rules. In Eqs. (25) and (26) by $\rho_{k}(s)$ we denote the nonperturbative contributions to $\rho^{Q C D}(s)$ and $g_{s}=4 \pi \alpha_{s}$. The explicit expressions for $\rho^{\text {pert }}(s)$ and $\rho_{k}(s)$ are presented below as the integrals over the Feynman parameters $z$ and $w$. Thus, for the diquark-antidiquark current we have found:

$$
\begin{aligned}
\rho^{\text {pert }}(s) & =\frac{1}{3072 \pi^{6}} \int_{0}^{1} d z \int_{0}^{1-z} d w \frac{w z}{h t^{8}}\left[s w z h-m_{c}^{2} t(w+z)\right]^{2} \\
& \times\left[35 h^{2} w^{2} z^{2} s^{2}-26 h t w z(w+z) s m_{c}^{2}\right. \\
& \left.+3 t^{2}(w+z)^{2} m_{c}^{2}\right] \theta[L],
\end{aligned}
$$

$$
\begin{aligned}
\rho^{3}(s) & =\frac{\langle\bar{u} u\rangle m_{c}}{64 \pi^{4}} \int_{0}^{1} d z \int_{0}^{1-z} d w \frac{\left[t(w+z) m_{c}^{2}-h w z s\right]}{t^{5}} \\
& \times(w+z)\left[7 s w z h-3 m_{c}^{2} t(w+z)\right] \theta[L]
\end{aligned}
$$




$$
\begin{aligned}
\rho^{4}(s) & =-\frac{1}{73728 \pi^{4}}\left\langle\alpha_{s} \frac{G^{2}}{\pi}\right\rangle \int_{0}^{1} d z \int_{0}^{1-z} d w \frac{w z}{h t^{6}} \\
& \times\left\{315 s^{2} h^{3} w^{3} z^{2}-3 m_{c}^{2} t^{2}(w+z)\left[71 w^{3}+3 w(z-1)\right.\right. \\
& \left.\times(23 z-10)+w^{2}(60 z-69)+2 z\left(15-30 z+31 z^{2}\right)\right] \\
& +2 m_{c}^{2} s h t w z\left[104 w^{3}+3 w(z-1)(17 z-25)-3 w^{2}\right. \\
& \left.\left.\times(31 z+17)+z\left(75-150 z+203 z^{2}\right)\right]\right\} \theta[L],
\end{aligned}
$$$$
\begin{aligned}
\rho^{5}(s) & =\frac{m_{c} m_{0}^{2}\langle\bar{u} u\rangle}{128 \pi^{4}} \int_{0}^{1} d z \int_{0}^{1-z} d w \frac{w z h(w+z)}{t^{5}} \\
& \times\left(20 s h w z-9 m_{c}^{2} t(w+z)\right) \theta[L],
\end{aligned}
$$

$$
\begin{aligned}
\rho^{6}(s) & =-\frac{\langle\bar{u} u\rangle^{2}}{12 \pi^{2}} \frac{\sqrt{s\left(s-4 m_{c}^{2}\right)}}{s}-\frac{g_{s}^{2}\langle\bar{u} u\rangle^{2}}{684 \pi^{4}} \int_{0}^{1} d z \int_{0}^{1-z} d w \\
& \times \frac{w z h^{2}\left[8 s h w z-3 m_{c}^{2}(w+z)\left(z^{2}+(w+z)(w-1)\right)\right]}{t^{5}} \theta[L],
\end{aligned}
$$

$$
\begin{aligned}
\rho^{7}(s) & =-\langle\bar{u} u\rangle\left\langle\alpha_{s} \frac{G^{2}}{\pi}\right\rangle \int_{0}^{1} d z \int_{0}^{1-z} d w \frac{m_{c}}{192 \pi^{2} t^{4}} \\
& \times \quad\left\{(w-1)\left[2 w^{4}-w z\left(w^{2}+z^{2}\right)\right]\right. \\
& \left.-\left[w^{3} z^{2}+(w-2) z^{4}+4 z^{2}+2 z^{6}\right]\right\} \theta[L] \\
& +\langle\bar{u} u\rangle\left\langle\alpha_{s} \frac{G^{2}}{\pi}\right\rangle \frac{m_{c} \sqrt{s\left(s-4 m_{c}^{2}\right)}}{384 \pi^{2} s}
\end{aligned}
$$$$
\rho^{8}(s)=-\frac{m_{c}^{2}}{13824 \pi^{2}}\left\langle\alpha_{s} \frac{G^{2}}{\pi}\right\rangle^{2} \int_{0}^{1} d z \int_{0}^{1-z} d w
$$$$
\times \quad \frac{w^{3}(w-1)+z^{3}(z-1)}{t^{2} h w z} \delta(s-\Phi)
$$$$
+\frac{m_{0}^{2}\langle\bar{u} u\rangle^{2} m_{c}^{2}}{24 \pi^{2}} \int_{0}^{1} d z\left[2 \delta\left(s-\frac{m_{c}^{2}}{z(1-z)}\right)\right.
$$$$
\left.+s \frac{d}{d s} \delta\left(s-\frac{m_{c}^{2}}{z(1-z)}\right)\right],
$$

$$
\begin{aligned}
\rho^{9}(s) & =-\frac{m_{c} m_{0}^{2}\langle\bar{u} u\rangle}{2304 \pi^{2}}\left\langle\alpha_{s} \frac{G^{2}}{\pi}\right\rangle \int_{0}^{1} d z \int_{0}^{1-z} d w \frac{1}{t^{3} h w z} \\
& \times\left\{3 h \left[2 w^{5}-w^{3} z(z-1)-w^{2} z^{3}-z^{3} w(z-1)\right.\right. \\
& \left.+2 z^{4}(z-1)-w^{4}(2+z)\right] \delta\left(s-\frac{m_{c}^{2}}{z(1-z)}\right) \\
& -\left[s h \left(w^{3}(2 w+5 h-2)-4 w^{4}(w-1)+2 w^{3} z^{2}\right.\right. \\
& \left.+w z^{3}(2 w+5 h-2)\right)-4 z^{5}+3 m_{c}^{2} t^{2}\left(w^{2}+z^{2}\right) \\
& \left.+2 z^{4}(2+w)\right] \frac{d}{d s} \delta\left(s-\frac{m_{c}^{2}}{z(1-z)}\right)-2 s\left(w^{2}+z^{2}\right) \\
& \left.\times s\left(m_{c}^{2} t^{2}+s w z h^{2}\right) \frac{d^{2}}{d s^{2}} \delta\left(s-\frac{m_{c}^{2}}{z(1-z)}\right)\right\},
\end{aligned}
$$$$
\begin{aligned}
\rho^{10}(s) & =\frac{g_{s}^{2}\langle\bar{u} u\rangle^{2}}{93312 \pi^{2}}\left\langle\alpha_{s} \frac{G^{2}}{\pi}\right\rangle\left\{\frac{8 m_{c}^{2}}{t^{2} w z} \int_{0}^{1} d z \int_{0}^{1-z} d w\right. \\
& \times \quad\left(w^{3}+z^{3}\right)\left[3 \frac{d}{d s} \delta(s-\Phi)+5 s \frac{d^{2}}{d s^{2}} \delta(s-\Phi)\right. \\
& \left.+s^{2} \frac{d^{3}}{d s^{3}} \delta(s-\Phi)\right] \\
& +\int_{0}^{1} d z \frac{1}{z}\left[\left(14 s z(z-1)-3 m_{c}^{2}\right) \frac{d}{d s} \delta\left(s-\frac{m_{c}^{2}}{z(1-z)}\right)\right. \\
& \left.\left.+2 s\left(m_{c}^{2}-2 s z(z-1)\right) \frac{d^{2}}{d s^{2}} \delta\left(s-\frac{m_{c}^{2}}{z(1-z)}\right)\right]\right\} . \quad(25)
\end{aligned}
$$

For the molecule current the results reads:

$$
\begin{aligned}
\rho^{\text {pert }}(s) & =\frac{1}{4096 \pi^{6}} \int_{0}^{1} d z \int_{0}^{1-z} d w \frac{w z}{h t^{8}}\left[s w z h-m_{c}^{2} t(w+z)\right]^{2} \\
& \times\left[4 m_{c}^{2} t(w+z)-7 s w z h\right]\left[3 m_{c}^{2} t(w+z)-5 s w z h\right] \theta[L], \\
\rho^{3}(s) & =\frac{3\langle\bar{u} u\rangle m_{c}}{256 \pi^{4}} \int_{0}^{1} d z \int_{0}^{1-z} d w \frac{\left[m_{c}^{2} t(w+z)-s w z h\right]}{t^{5}} \\
& \times(w+z)\left[7 s w z h-3 m_{c}^{2} t(w+z)\right] \theta[L], \\
\rho^{4}(s) & =\frac{1}{6144 \pi^{4}}\left\langle\alpha_{s} \frac{G^{2}}{\pi}\right\rangle \int_{0}^{1} d z \int_{0}^{1-z} d w \frac{w z}{h t^{6}}\left\{7 m_{c}^{2} s h t w z\right. \\
& \times(w+z)\left[5 w z-2 z^{2}+w(w-3)\right]-27 s^{2} h^{3} w^{3} z^{2} \\
& \left.+5 m_{c}^{4} t^{2}\left(w^{3}+z^{3}\right)(w+z)\right\} \theta[L],
\end{aligned}
$$

$$
\begin{aligned}
\rho^{5}(s)= & \frac{3 m_{c} m_{0}^{2}\langle\bar{u} u\rangle}{512 \pi^{4}} \int_{0}^{1} d z \int_{0}^{1-z} d w \frac{h(w+z)}{t^{4}} \\
& \times\left[5 s h w z-3 m_{c}^{2} t(w+z)\right] \theta[L], \\
\rho^{6}(s)= & -\frac{\langle\bar{u} u\rangle^{2} m_{c}^{2}}{16 \pi^{2}} \frac{\sqrt{s\left(s-4 m_{c}^{2}\right)}}{s}-\frac{g_{s}^{2}\langle\bar{u} u\rangle^{2}}{1728 \pi^{4}} \int_{0}^{1} d z \int_{0}^{1-z} d w \\
\times & \frac{w z h^{2}\left\{14 s h w z-5 m_{c}^{2}(w+z)\left[(w+z)(w-1)+z^{2}\right]\right\}}{t^{5}} \theta[L], \\
\rho^{7}(s)= & -\langle\bar{u} u\rangle\left\langle\alpha_{s} \frac{G^{2}}{\pi}\right\rangle \int_{0}^{1} d z \int_{0}^{1-z} d w \frac{m_{c}}{256 \pi^{2} t^{4}}\left[2 w^{5}-w z(z-1)\right. \\
\times & \left.\left(w^{2}+z^{2}\right)-w^{2} z^{3}+2 z^{4}(z-1)-w^{4}(z+2)\right] \theta[L] \\
+ & \langle\bar{u} u\rangle\left\langle\alpha_{s} \frac{G^{2}}{\pi}\right\rangle \frac{m_{c} \sqrt{s\left(s-4 m_{c}^{2}\right)}}{512 \pi^{2} s} \\
\rho^{8}(s)= & -\frac{m_{c}^{4}}{55296 \pi^{2}}\left\langle\alpha_{s} \frac{G^{2}}{\pi}\right\rangle^{2} \int_{0}^{1} d z \int_{0}^{1-z} d w \frac{h w^{2} z^{2}}{t^{2} h^{4}} \\
\times & {\left[3 \frac{d}{d s} \delta(s-\Phi)+5 s \frac{d^{2}}{d s^{2}} \delta(s-\Phi)-s^{2} t^{2} \frac{d^{3}}{d s^{3}} \delta(s-\Phi)\right] } \\
+ & \frac{m_{0}^{2}\langle\bar{u} u\rangle^{2} m_{c}^{2}}{32 \pi^{2}} \int_{0}^{1} d z\left[2 \delta\left(s-\frac{m_{c}^{2}}{z(1-z)}\right)\right. \\
+ & \left.s \frac{d}{d s} \delta\left(s-\frac{m_{c}^{2}}{z(1-z)}\right)\right],
\end{aligned}
$$

$$
\begin{aligned}
\rho^{9}(s) & =-\frac{m_{c} m_{0}^{2}\langle\bar{u} u\rangle}{3072 \pi^{2}}\left\langle\alpha_{s} \frac{G^{2}}{\pi}\right\rangle \int_{0}^{1} d z \int_{0}^{1-z} d w \frac{1}{t^{3} h w z} \\
& \times\left\{3 ( w + z - 1 ) \left[2 w^{5}-(z-1)\left(w^{3} z+w z^{3}\right)\right.\right. \\
& \left.-w^{2} z^{3}+2 z^{4}(z-1)-w^{4}(2+z)\right] \delta(s-\Phi) \\
& -\left[s ( w + z - 1 ) \left((w-1)\left(4 w^{4}-7 w^{3} z-7 w z^{3}\right)\right.\right. \\
& \left.\left.-z^{4}(4+7 w)+4 z^{5}\right)+3 m_{c}^{2} t^{2}\left(w^{2}+z^{2}\right)\right] \frac{d}{d s} \delta(s-\Phi) \\
& \left.+2 s\left(w^{2}+z^{2}\right)\left(m_{c}^{2} t^{2}+s h^{2} w z\right) \frac{d^{2}}{d s^{2}} \delta(s-\Phi)\right\}, \\
\rho^{10}(s) & =\frac{g_{s}^{2}\langle\bar{u} u\rangle^{2}}{31104 \pi^{2}}\left\langle\alpha_{s} \frac{G^{2}}{\pi}\right\rangle\left\{2 m_{c}^{2} \int_{0}^{1} d z \int_{0}^{1-z} d w \frac{w^{3}+z^{3}}{t^{2} w z}\right. \\
& \times\left[3 \frac{d}{d s} \delta(s-\Phi)+5 s \frac{d^{2}}{d s^{2}} \delta(s-\Phi)+s^{2} \frac{d^{3}}{d s^{3}} \delta(s-\Phi)\right] \\
+ & s \int_{0}^{1} d z(z-1)\left[7 \frac{d}{d s} \delta\left(s-\frac{m_{c}^{2}}{z(1-z)}\right)\right. \\
& \left.\left.-2 s \frac{d^{2}}{d s^{2}} \delta\left(s-\frac{m_{c}^{2}}{z(1-z)}\right)\right]\right\} .
\end{aligned}
$$


In the expression above we have used the notations:

$$
\begin{aligned}
L & =-\frac{(w-1)\left[m_{c}^{2}\left(w^{3}+w^{2}(2 z-1)+\left(z^{2}+2 w z\right)(z-1)\right)-s w z h\right]}{t^{2}} \\
\Phi & =\frac{m_{c}^{2}\left[w^{3}+z(z-1)(2 w+z)+w^{2}(2 z-1)\right]}{w z h} \\
t & =w^{2}+(z-1)(w+z), \\
h & =w+z-1 .
\end{aligned}
$$

